Cahiers $d u$ MONDE RUSSE

\section{Cahiers du monde russe}

Russie - Empire russe - Union soviétique et États indépendants

$43 / 4 \mid 2002$

Intellectuels et intelligentsia

\title{
N. E. Koposov, Kak dumajut istoriki
}

\section{Antonella Salomoni}

\section{OpenEdition \\ Journals}

Édition électronique

URL : https://journals.openedition.org/monderusse/4062

DOI : $10.4000 /$ monderusse. 4062

ISSN : $1777-5388$

\section{Éditeur}

Éditions de l'EHESS

\section{Édition imprimée}

Date de publication : 30 décembre 2002

Pagination : 811-813

ISBN : 2-7132-1796-2

ISSN : $1252-6576$

\section{Référence électronique}

Antonella Salomoni, «N. E. Koposov, Kak dumajut istoriki », Cahiers du monde russe [En ligne], 43/4 | 2002, mis en ligne le 06 juillet 2009, consulté le 03 septembre 2022. URL : http://

journals.openedition.org/monderusse/4062 ; DOI : https://doi.org/10.4000/monderusse.4062

Ce document a été généré automatiquement le 3 septembre 2022

Tous droits réservés 


\title{
N. E. Koposov, Kak dumajut istoriki
}

\author{
Antonella Salomoni
}

\section{RÉFÉRENCE}

N. E. KOPOSOV, Kak dumajut istoriki (Comment pensent les historiens), Moscou, Novoe literaturnoe obozrenie, 2001, $326 \mathrm{p}$.

1 Les interrogations sur la façon de travailler des historiens et sur les représentations qu'ils offrent du passé constituent, depuis quelque temps, un centre d'intérêts renouvelés aussi bien à l'intérieur qu'à l'extérieur de la discipline en dépit de l'extrême hétérogénéité des fins poursuivies par les chercheurs. Le dernier livre de N.E. Koposov se distingue du grand nombre des ouvrages à visée critique qui ont vu le jour au cours des dernières années par son ambition de comprendre non seulement la façon de travailler des historiens et leurs représentations, mais aussi la pensée qui gouverne l'esprit du chercheur et crée les images de la société.

Une approche importante de la question est issue des théories considérant les représentations de la réalité comme des protocoles. Le linguistic turn, utilisé avec discernement, permet d'approcher l'esprit de l'historien, étant donné que l'histoire est accessible sous une forme surtout linguistique, mais cette dernière s'impose aussi à l'intellection par des modalités différentes de l'expression verbale. Lorqu'il s'agit de décrire les structures sociales du passé, les différentes modalités de représentation interne - linguistiques et non linguistiques - entrent en coopération. Le livre de Koposov prend en considération l'historiographie française des "ordres" et des "classes ", posant au centre de son analyse le fait que - au cours des années 1960 - les historiens sociaux, dans la construction de leurs représentations de la stratification, ont eu recours à des procédures intellectuelles inadéquates, et parfois erronées, au point de provoquer une crise de la discipline. L'historien, d'une part, s'appliquait à saisir la signification des «noms» imposés ou assignés aux groupes sociaux qu'il devait examiner (opération linguistique) ; de l'autre, il devait disposer et faire agir dans son esprit des objets, c'est-àdire des individus, sans «nom » à travers lesquels il tentait de dresser un modèle de 
société (organisation empirique). Le processus de construction inductive des groupes sociaux a mis au jour des agrégations qui n'avaient pas d'entrées dans le vocabulaire reçu par la tradition. Le fait est que les catégories du premier champ et celles du deuxième répondent à des logiques de type différent. Mettre ensemble des individus en ayant recours à une procédure empirique comme la classification (distribution dans une catégorie selon des critères déterminés) amène à obtenir une "multitude». Tant que l'histoire sociale se réduisait à une herméneutique des termes sociaux, la contradiction est demeurée latente. Mais lorsque, pour des raisons inhérentes à l'application systématique des sciences auxiliaires ou limitrophes, l'histoire sociale a abandonné l'interprétation des significations, il y a eu déflagration ou crise. Le débat sur les « ordres » et les « classes » en est l'un des effets.

3 Le premier chapitre du livre place au centre de l'analyse la question des « ordres » et des « classes » ainsi qu'elle a été abordée dans les études des historiens français des années 1960 aux prises avec la société d'Ancien Régime. Le titre («Herméneutique et classification ») est en soi plutôt éloquent : la pluralité ou le spectre des interprétations, dérivant des différentes positions culturelles et idéologiques des chercheurs, est supporté par une structure logique identique, visant à reconstruire entièrement la hiérarchie sociale à travers la classification empirique des individus qui la constitue. Mais il s'agit de procédures mentales inconciliables. La crise de l'histoire sociale n'aurait donc pas seulement des motivations d'ordre idéologique, il faudrait aussi l'imputer au fait que les critères adoptés étaient intimement en conflit.

4 L'auteur avance une démonstration dans le deuxième chapitre («Sémantique des catégories sociales ») en examinant, à partir de John Stuart Mill, les principales théories de la signification face à celles de la classification. Il propose une vision sémantique des catégories sociales d'après laquelle les représentations mentales que nous en avons sont constituées de deux ensembles opposés que l'on peut difficilement transmuter l'un dans l'autre, compte tenu de leur nature différente : l'un qui reçoit les connotations (les êtres sont connus à travers des caractères déterminés et nous sommes informés sur certaines de leurs prérogatives individuelles) ; l'autre qui accueille une image de compréhension totale désignant les propriétés de la classe d'appartenance.

5 Le troisième chapitre (" Naissance de la société à partir de la logique de l'espace »), ainsi que le quatrième («Névrose de la classification») s'interrogent en particulier sur les influences possibles exercées par la logique de l'espace dans la constitution et la pratique de l'histoire sociale. La généalogie proposée ici est la suivante: la notion grecque de politeia se désintègre en concomitance avec le processus de formation de l'État moderne occidental et de la société européenne moderne ; l'idée de société, telle qu'elle vient se structurer au XVIII ${ }^{e}$ siècle, dérive de l'image de multitude, qui est cependant un dispositif paralogique ; l'image de la hiérarchie sociale d'Ancien Régime est une vision générale qui résulte d'un acte mental réunissant différentes représentations ; les illustrations du social sont les effets, dans le premier âge moderne, de la révolution qui se produit dans la perception de l'espace aussi bien dans les sciences que dans les arts plastiques. Ces éléments, on peut les retrouver dans la structure logique de l'histoire sociale des années 1960. Dans le passage du premier âge moderne (avec son legs de la Renaissance à la peinture) au deuxième, on retrouve des déplacements ultérieurs de la notion de social et une sorte d'hypertrophie de l'image spatiale de l'histoire qui arrive jusqu'à la période du positivisme, lorsque l'avènement de l'histoire sociale va se placer dans le cadre de l'image stratifiée de l'histoire, à côté de toutes les autres histoires "particulières " 
(économique, politique, culturelle). Mais, avec l'entrée dans la contemporanéité, on va abandonner la référence à l'espace et s'entrouvre alors une nouvelle dimension qui permet à l'auteur d'étudier de manière approfondie l'approche constructiviste et les grands courants de critique de la raison historique.

6 Koposov considère - comme il l'explique dans les conclusions de son travail (« De la culture au sujet »)- que les sciences sociales abordent encore avec beaucoup de difficultés l'étude des dispositions mentales des chercheurs, car elles restent comme prisonnières de la conception qu'elles en avaient au moment de leur constitution en discipline. Ce livre important est un premier pas en direction d'une compréhension de la mentalité des historiens. 